\title{
AUTOMATIC 4-D REGISTRATION IN DYNAMIC MR RENOGRAPHY
}

\author{
${ }^{1}$ Ting Song, ${ }^{2}$ Vivian S. Lee, ${ }^{2}$ Henry Rusinek, ${ }^{2}$ Manmeen Kaur, ${ }^{1}$ Andrew F. Laine
}

${ }^{1}$ Department of Biomedical Engineering, Columbia University, New York, NY, U.S.A.

${ }^{2}$ Department of Radiology, MRI, New York University Medical Center, New York, NY, U.S.A.

\section{INTRODUCTION}

\section{Dynamic contrast-enhanced 3D MR renography has the} potential for broad clinical applications. Although high quality images can be acquired during repeated breathholds, manual registration and segmentation of serially acquired images of the kidney is prohibitively laborintensive.

- Our purpose was to develop automatic registration and segmentation algorithms and to test its effectiveness.

- The framework was firstly evaluated on two 3D MR renography datasets that simulated respiratory motion, representative of patterns of contrast enhancement in a

- The proposed algorithm was also evaluated on simulatec motion data derived from a real patient data set, and tested on 12 clinical patient data sets.

\section{JЕЈHODOLOGY}

Crop a 4D volume containing a single kidney

$$
\text { Anisotropic Diffusion }
$$

Extract edge information of the $4 \mathrm{D}$ volume

Subvoxel Registration

Principal Component Analysis

Use k-means clustering to collecting system

2.1 Anisotropic Diffusion and Wavelet Edge Detection

- An edge-preserving anisotropic diffusion algorithm was applied to suppress noise (Figs 1 and 2).

n Due to the perfusion process, using intensity images directly is unreliable. A 3D over-complete dyadic wavele transform was applied to extract "edge" information. We selected the modulus at level 2 for the registration.

Fig. 1. The effect of

anisotropic diffusion

Fig. 2. Comp

methods.

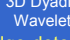

\subsection{Fourier-Based Registration}

- Let $f(x)$ be a 3D object and let $g(x)$ be a rigidly translated and rotated version of $f(x)$. It can be shown that $f(x)$ relates to $g(x)$ as (1) and that Fourier transforms are related as (2). The translational vector $t$ affects only phase and not magnitude values. Magnitudes are related as (3).

$f(\mathrm{X}), \mathrm{X} \in \mathbb{R}^{3}$

$g(\mathrm{X})=f\left(R^{-1} \mathrm{X}-t\right), t \in \mathbb{R}^{3}, R \in \mathbb{R}^{33}$

$G(\mathrm{~K})=F\left(R^{-1} \mathrm{~K}\right) e^{-j 2 K^{T} / R}$ (2) $|G(\mathrm{~K})|=\left|F\left(R^{-1} \mathrm{~K}\right)\right|$

\subsection{Subvoxel Registration}

- A subvoxel registration step was introduced as a refining process toward a more accurate registration result. With the Fourier Transform, the problem was converted into a 3D plane fitting problem in 3D frequency domain, which was solved by least squared fitting.

2.4 PCA and K-Means Clustering

- Signal intensities along the time dimension were used to do the principal component analysis (PCA). After PCA K-Means clustering was used to segment (or classify) the kidney into three distinct clusters corresponding to the cortex, medulla and collecting system. normal and dysfunctional kidney.

Estimate and correct the rotation and translation

segment the cortex, medulla and

\section{METHODOLOGY (COnt)}
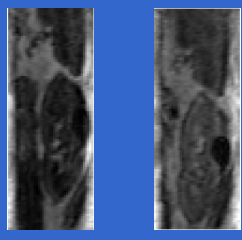

$1^{\text {st }}$ frame

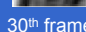

$30^{\text {th }}$ frame
(without rotation

correction)

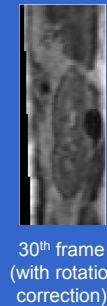

Fig. 3. Illustration of the rotation problem.

\section{EVALUATION}

\subsection{Simulated Phantom Study}

- Based on a simulated data set, a phantom with simulated respiratory motion was created by translating and rotating the kidney, representative of typical motion seen with a poorly-compliant patient.

n Simulated motions included head to feet (HF) translation left to right (LR) translation, anterior to posterior (AP) translation, and rotation (Rot) with respect to three different axes, which were defined by the axis of rotation and the angle of the rotation along that axis.

n In the normal (NL) functioning kidney we simulated concentration-time curves in each renal compartmen (cortex, medulla, collecting system) that were representative of normal kidneys with glomerular filtration rate (GFR) equal $60 \mathrm{ml} / \mathrm{min}$. In the second abnormally (ABN) functioning kidney, we simulated a GFR=30 ml/min

\subsection{Clinical Phantom Study}

- A similar set of data was simulated by introducing translation and rotation into a patient data set with dimension [779740 20] and voxel resolution $1.66 \mathrm{~mm}$ x $1.66 \mathrm{~mm} \times 2.5 \mathrm{~mm}$.

\subsection{Clinical Evaluation}

- Our algorithm was applied to 12 clinical patients datasets (24 kidney in total), with manual registration and segmentation serving as ground truth.

- All data sets consisted of at least 41 3D acquisitions, where each 3D dataset was similar to the clinical phantom data set.

n Time-intensity curves of cortex, medulla, and collecting systems were calculated based on both our automatic registration method and a Mutual Information (MI) approach were compared to ground truth.

\section{RESULTS}

4.1 Simulated Phantom Study

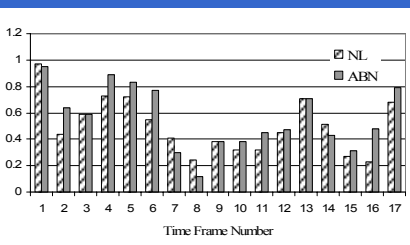

Fig. 4. Errors of rotation correction in the units of degree.

- Across 18 time frames, the average ( \pm st.dev) error of rotation was $0.50 \pm 0.21$ degrees for NL and $0.56 \pm$ 0.24 degrees for $\mathrm{ABN}$ (Fig 4).

4 PCA and K-Means Clustering
Signal intensities along the time dimension were used to
do the principal component analysis (PCA). After PCA,
K-Ilistering was used to segment (or classify)
the clusters corresponding to

\section{RESULTS (Cont)}

- Representative segmentation results of NL case are shown in Fig 5. The root-mean-square (RMS) errors in time-signal intensity curves for the NL were $1.7 \%, 6.9 \%$, and $3.7 \%$ corresponding to cortex, medulla and collecting system; for the ABN, the RMS were : $2.6 \%, 10.9 \%$, and $8.6 \%$, respectively.

4.2 Clinical Phantom Study

- Translation estimation errors were $0.53 \pm 0.47,0.51 \pm 0.46$, and $0.60 \pm 0.41$ in $x, y$, and $z$ direction respectively; errors in rotation angles were less than 2.5 degrees with mean value $0.003 \pm 0.003,0.07 \pm 0.26$, and $1.14 \pm 0.72$ degrees in $(\Theta, \Phi, \Psi)$.
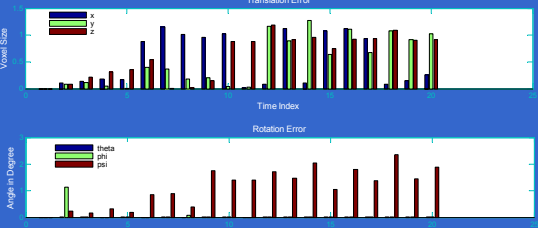

Fig. 6. Clinical phantom study results.

\subsection{Clinical Evaluation}

- The average relative errors for cortex, medulla and collecting systems in our method were $3.24 \% \pm 1.41 \%$ $5.31 \% \pm 2.19 \%$, and $8.23 \% \pm 3.35 \%$ respectively for the left and $3.99 \% \pm 2.23 \%, 5.67 \% \pm 4.13 \%$, and $9.26 \% \pm 5.94 \%$ for the right.
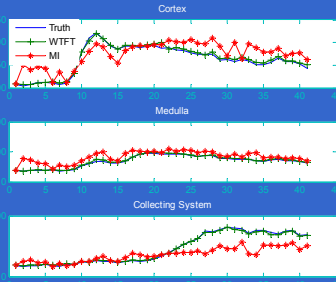

Fig. 7. Average intensity curves for one of the data sets usin manual registration, WTFT, and MI.

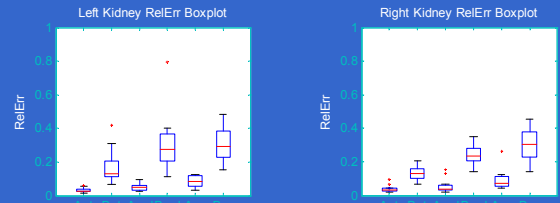

Fig. 8. Box plot for RMS evaluation of the time-intensity curves generated from WTFT (A) and MI (B) methods. 'ct', cortex; 'md', medulla; 'cs', collecting system. (a) left kidneys, (b) right kidneys.

5. SUMJMVARY \&: CONCLUSION

- We have employed new methods for 3D MR renography image registration including anisotropic diffusion as denoising method and 3D overcomplete wavelet transform. Subvoxel registration was also incorporated.

- Our automatic registration and segmentation algorithms showed accurate results when compared to manual registration by expert radiologists on simulated and representative clinical 3D MR renography datasets.

\section{REFERRENCES}

[1] V. S. Lee, H. Rusinek, M. E. Noz, P. Lee, M. Raghavan, and E. L. Kramer, "Dynamic Three-dimensional MR Renography for the Measurement of Single Kidney Function: Initial Experience," Radiology, vol. 227, pp. 289-94, 2003.

[2] Q. Duan, et al., "Assessment of visual quality and spatial accuracy of fast anisotropic diffusion and scan conversion algorithms for realtime three-dimensional spherical ultrasound," Proc. SPIE, Vol. 5373 pp. 331-42, 2004. 